\title{
Erratum to: Testosterone stimulates glucose uptake and GLUT4 translocation through LKB1/AMPK signaling in 3T3-L1 adipocytes
}

\author{
Kazuteru Mitsuhashi ${ }^{1}$ - Takafumi Senmaru ${ }^{1}$ - Takuya Fukuda ${ }^{1}$ - Masahiro Yamazaki $^{1}$. \\ Katsuhiko Shinomiya $^{2}$ - Morio Ueno ${ }^{2}$ Shigeru Kinoshita ${ }^{2} \cdot$ Jo Kitawaki $^{3}$. \\ Masato Katsuyama $^{4} \cdot$ Muneo Tsujikawa $^{5} \cdot$ Hiroshi Obayashi ${ }^{5} \cdot$ Naoto Nakamura $^{1}$. \\ Michiaki Fukui ${ }^{1}$
}

Published online: 28 January 2016

(C) Springer Science+Business Media New York 2016

\section{Erratum to: Endocrine (2016) 51:174-184 \\ DOI 10.1007/s12020-015-0666-y}

Unfortunately, the authors found that the image of the loading control of the Western blot of AMPK which was used in Fig. 2b was incorrect in the published version. Figure 2 has been corrected with this erratum.

The online version of the original article can be found under doi:10.1007/s12020-015-0666-y.

\section{Takafumi Senmaru}

semmarut@koto.kpu-m.ac.jp

1 Department of Endocrinology and Metabolism, Kyoto Prefectural University of Medicine, Graduate School of Medical Science, 465 Kajii-cho, Kawaramachi-Hirokoji, Kamigyo-ku, Kyoto 602-8566, Japan

2 Department of Ophthalmology, Kyoto Prefectural University of Medicine, Graduate School of Medical Science, Kyoto, Japan

3 Department of Obstetrics and Gynecology, Kyoto Prefectural University of Medicine, Graduate School of Medical Science, Kyoto, Japan

4 Radioisotope Center, Kyoto Prefectural University of Medicine, Kyoto, Japan

5 Institute of Bio-Response Informatics, Kyoto, Japan 
(a)

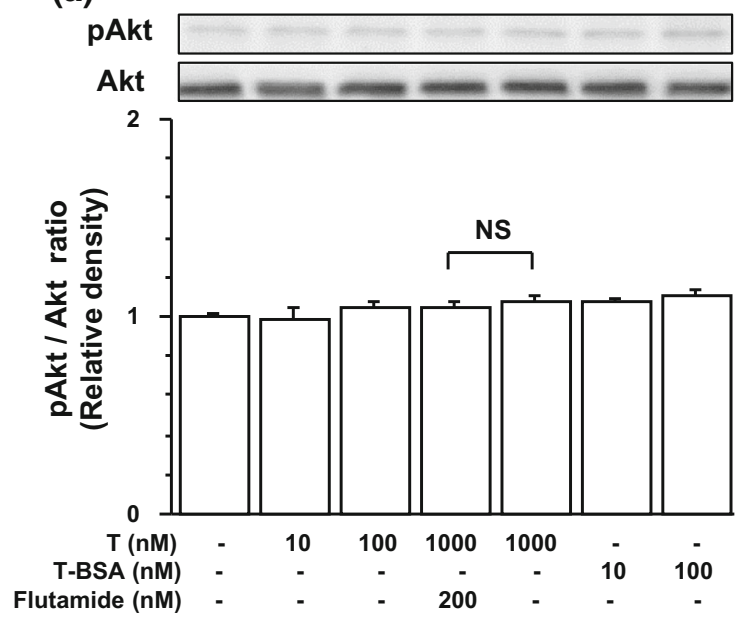

(b)

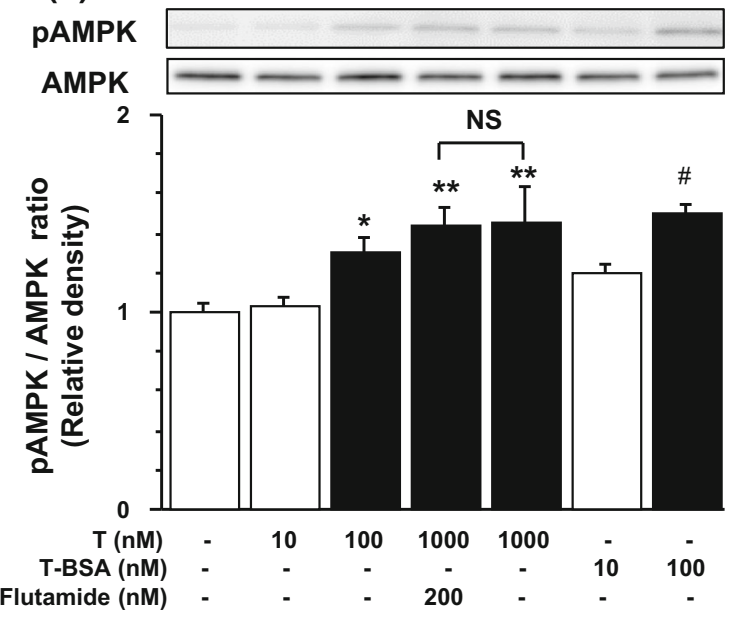

Fig. 2 Effects of testosterone and testosterone-BSA on phosphorylation of Akt, AMPK, LKB1, and CaMKI. 3T3-L1 adipocytes were incubated with testosterone or testosterone-BSA at the indicated concentrations with or without pre-incubation with flutamide $(200 \mathrm{nM})$ for $2 \mathrm{~h}$. Western blot analysis with anti-Akt and anti- (c)

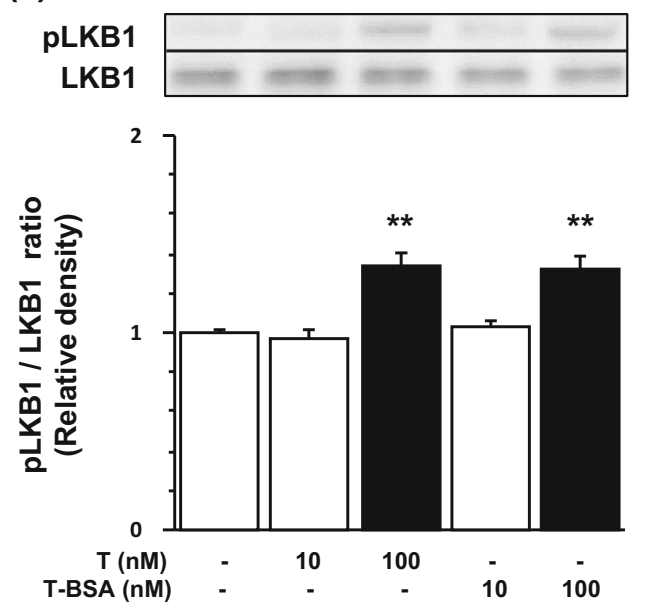

(d)
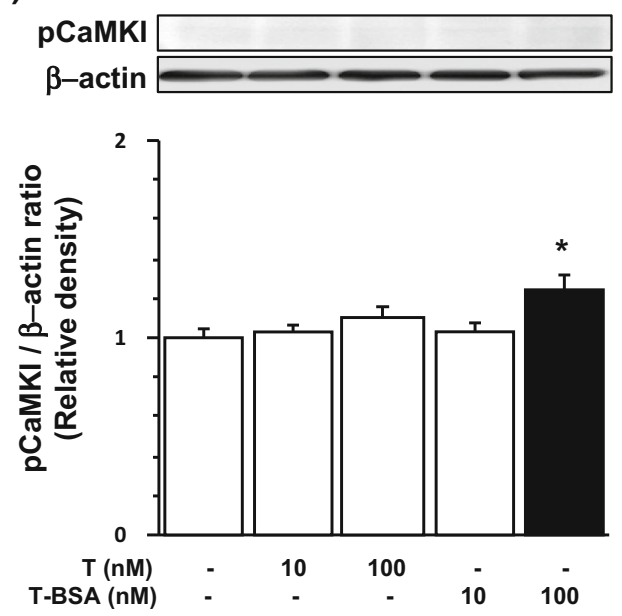

phospho-Akt $\left(\operatorname{Ser}^{473}\right)$ (a), anti-AMPK and anti-phospho-AMPK $\left(\mathrm{Thr}^{172}\right)(\mathbf{b})$, anti-LKB1 and anti-phospho-LKB1 $\left(\mathrm{Ser}^{428}\right)(\mathbf{c})$, and anti- $\beta$-actin and anti-phospho-CaMKI $\left(\mathrm{Thr}^{177}\right)(\mathbf{d})$. Values are given as mean \pm SEM of four independent experiments. $* P<0.05$ and $* * P<0.01$ versus non-treated cells. NS not significant 